Meta

Journal des traducteurs

Translators' Journal

\title{
Mon expérience de traducteur
}

\section{José María Valverde}

Volume 38, numéro 4, décembre 1993

Le Je du traducteur

The $I$ of the Translator

URI : https://id.erudit.org/iderudit/004530ar

DOI : https://doi.org/10.7202/004530ar

Aller au sommaire du numéro

Éditeur(s)

Les Presses de l'Université de Montréal

ISSN

0026-0452 (imprimé)

Découvrir la revue

Citer cet article

Valverde, J. (1993). Mon expérience de traducteur. Meta, 38(4), 603-614. https://doi.org/10.7202/004530ar d'utilisation que vous pouvez consulter en ligne.

https://apropos.erudit.org/fr/usagers/politique-dutilisation/ 


\title{
MON EXPÉRIENCE DE TRADUCTEUR'
}

\author{
JOSÉ MARÍA VALVERDE \\ Université de Barcelone, Barcelone, Espagne
}

Mon sujet ne peut être la théorie de la traduction, car je n'ai pas de théorie et, qui plus est, je ne crois pas en la théorie de la traduction. La traduction est pour moi un métier, une activité imitative. C'est comme ce travail que font les acteurs comiques qui imitent les hommes politiques et tentent de reproduire leur voix. Moi je fais de même: j'écoute l'auteur et j'essaie d'en conserver la voix. Cela suppose non seulement d'avoir l'oreille, mais encore de renoncer à soi. Traduire est une activité d'une grande valeur morale, parce que c'est un exercice ascétique dans un double sens: d'abord parce qu'on le fait toujours mal - c'est donc un bon exercice de formation du caractère; en second lieu, parce qu'il faut s'oublier soi-même. Si le traducteur a un style littéraire qui lui est propre, il doit l'oublier complètement: les stylistes sont incapables de traduire. C'est pour cette raison que, pour l'écrivain - et c'est mon cas —, traduire a un bon côté mais aussi un mauvais. Le bon, c'est que cette activité l'oblige à rompre avec les clichés, à être prêt à tout, à se montrer souple et à être toujours ouvert à n'importe quelle forme; l'inconvénient, c'est qu'on peut arriver à trop omettre ces clichés qui, en fin de compte, sont nécessaires et l'on court alors le danger d'avoir un style par trop incolore. Ceci serait grosso modo l'aspect éthique de la traduction, lequel n'est pas éthique par rapport au traduire mais par rapport à soi-même : la traduction comme exercice moral, comme éducation personnelle.

Pour ma part, j'ai beaucoup traduit. Si l'on empilait les livres que j'ai traduits tout au long de ma vie, la pile serait plus haute que moi. J'ai traduit essentiellement des ouvrages de langues anglaise et allemande, mais aussi du français et de l'italien. En réalité, l'italien est ma seconde langue, mais on ne me propose pas souvent de traduire dans cette langue. D'ailleurs, les traductions italiennes sont moins bien payées; cet aspect a aussi son importance. Enfin, cela ne me plaît pas autant. Ce qui a pour moi de l'attrait, c'est traduire de l'allemand, quand il s'agit de vers et, quand il s'agit de prose, de l'anglais. Les raisons n'en sont pas très claires pour moi. Peut-être est-ce parce qu'en allemand il faut moduler une plus grande distance linguistique, ce qui me permet de mieux rendre la forme poétique avec mon parti-pris de traducteur littéral, totalement littéral. En prose, au contraire, c'est l'anglais que je préfère, parce que la prose anglaise est meilleure que la prose allemande. A ce sujet, il y a des opinions divergentes, mais, personnellement, je crois que ce sont les Anglais qui écrivent la meilleure prose. Ils écrivent également bien en vers. Mais là, j'ai une gamme plus réduite de possibilités, entre autres raisons, parce que, si l'on veut faire une version versifiée, il est très difficile de procéder en passant de l'anglais à l'espagnol, car il y a toujours des syllabes en trop. Onze syllabes en anglais donnent treize ou quatorze ou quinze syllabes en espagnol. En allemand, il y a aussi des syllabes en trop, mais elles ne sont pas en si grand nombre: il est possible de trouver des solutions en passant de l'hendécasyllabe à l'alexandrin. En anglais, c'est chose impossible.

J'ai commencé à traduire quand j'étais adolescent et j'ai découvert Rilke. Je lisais mal l'allemand. J'avais été un an ou deux au Collège allemand, mais j'y avais beaucoup souffert et la seule chose que j'y avais apprise était à chanter un peu dans cette langue. J'avais assimilé la phonétique et guère plus. Il régnait dans cette école une atmosphère de barbarie incroyable. Plus tard, je me rendis compte que nous étions dans les années 1933-34, 
celles de Hiltler. Le fait est qu'à partir de ce moment, j'ai pu lire un peu en allemand. C'est à dix-sept ans que j'ai découvert Rilke, et je me suis aperçu que ses vers devaient être lus en allemand mais, en même temps, que je devais les traduire pour les posséder réellement. Je me suis donc mis à les traduire et $\mathrm{j}$ 'ai même donné forme à ces versions que j'ai réunies peu à peu en un ensemble. En 1957, j'ai publié un premier tome de ces traductions intitulé Cinquante poèmes de Rilke, grâce auquel j'ai obtenu le Prix National Fray Luis de León, qui m'a été décerné deux fois. La seconde fois, ce fut pour la traduction d'Ulysse de Joyce. Etant donné qu'il s'agissait à la fois d'une langue et d'une forme différente, cet hommage me paraît légitime.

Quelque temps plus tard, j'ai fait une version augmentée des Cinquante poèmes de Rilke, parce que José Janés m'avait demandé de préparer un grand volume sur cet auteur. J'y ai inclus Les Cahiers de Malte. L'éditeur voulait que je traduise dix mille vers de Rilke. Dix mille vers, c'est énorme, mais, les impératifs commerciaux étant ce qu'ils sont, je l'ai fait. Janés est mort pendant que je réalisais ce travail, et on a intégré le volume dans une autre collection. L'intention était de l'augmenter encore. C'est alors que j'ai suggéré d'ajouter le texte allemand en regard. Voilà qui était embarrassant pour moi, car j'avais traduit sans penser que le texte original figurerait en regard et, de cette manière, je m'étais permis d'être moins fidèle. En fin de compte, il me fallut offrir le texte original allemand, chose dont je me réjouissais néanmoins: on verrait ainsi que ma traduction était exacte. Le problème avec Rilke, c'est qu'il a utilisé le mètre et la rime, sauf dans les Élégies de Duino (que j'ai reprises récemment aux Éditions Lumen mais qui, pour moi, traducteur, m'ont semblé offrir une gamme de possibilités réduite, parce qu'il est plus facile de traduire les Élégies que les livres antérieurs dans lesquels il y a mètre et rime). Pour ma part, je n'ai pas conservé la rime, mais seulement le mètre. Toutefois, dans certains cas, j'ai utilisé l'alexandrin au lieu de l'hendécasyllabe. Voici, par exemple, le premier poème de l'ensemble, dans un vers qui n'est pas celui de la version originale. Celui-ci était de huit syllabes et j'ai utilisé celui de neuf (rappelons qu'en allemand le vers énéasyllabe n'existe pas). Le poème a pour titre «Le poète»:

\section{Der Dichter}

Du entfernst dich von mir, du Stunde. Wunden schlägt mir dein Flügelschlag. Allein: was soll ich mit meinem Munde? mit meiner Nacht? mit meinem Tag?

Ich habe keine Geliebte, kein Haus, keine Stelle auf der ich lebe.

Alle Dinge, an die ich mich gebe, werden reich und geben mich aus.

\section{El poeta}

Hora, te alejas ya de mí. Me hiere el golpe de tus alas. Solo, ¿qué haré yo con mi boca? ¿Y con mi noche? ¿Y con mi dia?

No tengo ni amada, ni casa; no tengo sitio donde habite. Las cosas, a las que me entrego, se enriquencen y me disipan.

Rilke est un poète extraordinaire et dont la voix est magnifique. J'ai essayé de l'imiter, mais la traduction n'est pas aussi belle que l'original.

Reculons dans le temps. Quand je terminais mes études de philosophie en 1947 ou 1948, j'ai découvert que la question du langage était concrètement posée chez le père de cette discipline, le grand romantique allemand Wilhelm von Humboldt, et je me proposai de faire ma thèse de doctorat - ce que j'ai fait - sur la philosophie du langage. Dans ce but, j'ai traduit, pour mon propre compte, le volumineux ouvrage de Humboldt sur la diversité de la structure linguistique, traduction que je n'ai pas publiée. En revanche, j'ai publié ma thèse, sous une forme quelque peu réduite, avec une petite anthologie des textes de Humboldt. Traduire est devenu pour moi, comme chez Humboldt lui-même et dans l'histoire de la linguistique, le point de départ de la prise de conscience de ce qu'est le langage, le centre de mes préoccupations culturelles en tant que professeur, voire 
comme poète. J'ai ensuite décidé de ne plus jamais traduire. Mais, plus tard, j'ai cédé de nouveau, en partie pour des raisons économiques. À partir de 1965, et pendant une période de deux ans, j'ai vécu uniquement de la traduction; à d'autres occasions, je l'ai faite comme activité complémentaire et parfois simplement par goût. En 1958 ou 1959, je me suis mis à traduire des textes théologiques allemands à la demande d'un éditeur, celui de la maison Guadarrama, qui devint plus tard Cristiandad. Je devins également le «traducteur» de Romano Guardini, bien que j'aie ignoré longtemps cette «clause» qui apparaissait dans le contrat. Celle-ci spécifiait que Guardini autorisait l'édition espagnole de ses ouvres à condition que j'en sois le traducteur exclusif. Guardini est un auteur très facile à traduire; en revanche j'ai traduit quelques textes de Urs von Baltasar, lesquels sont difficiles de compréhension même pour les Allemands. Baltazar invente un terrible langage. Dans le même genre, paraitront bientôt les essais de Heiddeger sur Hölderlin, qui sont la chose la plus difficile à traduire que j'aie jamais rencontrée.

J'ai eu aussi, un jour, l'idée de traduire les Évangiles uniquement pour mon plaisir, parce que j'en avais assez des traductions qui avaient cours. Cela se passait avant Vatican II, à un moment où la liturgie était encore en latin et où les traductions étaient abominables. Au moment où je terminais ma traduction, l'éditeur de Guardini avait été informé de ce que je faisais et il me demanda de lui faire lire ma traduction. Elle fut révisée par un spécialiste de l'araméen, qui m'aida pour les araméismes et les hébraïsmes, et je l'ai publiée, non sans craintes. En effet, qu'un laïc se lance à ce moment-là dans le projet de proposer une nouvelle version des Évangiles pouvait paraître audacieux. Cependant, personne n'y trouva rien à redire. Quelque temps plus tard, le Concile eut lieu et la liturgie adopta la langue vulgaire. Je fus appelé par la commission liturgique, où j'avais de très bons amis (parmi eux, l'actuel doyen de l'Institut Biblique de Rome, le jésuite Luis Alonso Schökel), pour collaborer à ses travaux. Et c'est ainsi que, pendant quelque temps, les lectionnaires s'appuyèrent sur les textes évangéliques que j'avais traduits. Je suis aussi intervenu en ce qui concerne les épîtres, et même la liturgie. Je me souviens qu'un jour, nous étions une vingtaine de personnes plongées dans la traduction du Canon de la messe, nous en avions tourné et retourné les phrases toute une journée. Un fait comique se produisit : à un moment donné, devant une énumération de noms de saints (Cécile, Lucie, Agnès, etc.), on se mit à discuter si on pouvait dire «Agata» (Agathe) puisque que «Agata» a une forme espagnole qui est «Agueda». Jimena Menéndez Pidal fit la suggestion suivante: «Comme il s'agit du Canon, je crois qu'on pourrait mettre Agatha Christi ! »

C'est ainsi que j'ai eu l'honneur d'intervenir jusque dans la formule de consécration de la messe, puisque la tendance des traductions antérieures, qui figuraient à titre de lectures personnelles, était de dire: «Celui-ci est mon corps»; j'ai pour ma part imposé la formule «Ceci est mon corps». Quelque chose de beau se produisit aussi alors que nous avions déjà les épreuves d'imprimerie du lectionnaire, tous les Américains du Sud - à l'exception des Argentins - prétendirent l'utiliser eux aussi. Cela posait un problème de changements grammaticaux. À une réunion à laquelle assistèrent deux ou trois Sud-Américains, un vote eut lieu. Les Andalous, les Sud-Américains et moi-même, qui suis d'Extramadoure, nous avons pris parti, contre l'avis du directeur, qui était de Valladolid, et de l'ensemble des Espagnols, en faveur de l'option syntaxique : «lo crucificaron» (ils le crucifièrent), rejetant «le crucificaron ${ }^{2} »$. Ce qu'en revanche nous n'avons pas admis, c'est le «Ustedes ${ }^{3}$ » parce que, pour les Espagnols, la différence était trop grande. Dans ce cas, il s'agissait en fait d'une traduction fonctionnelle, réalisée à des fins pratiques. Par la suite, quelques années plus tard, je suis intervenu dans l'édition d'une Nouvelle Bible Espagnole, bien qu'uniquement à titre de réviseur littéraire de la section hébraïque, ce qui ne laisse pas d'être curieux puisque j'avais fait, de mon côté, la traduction de la partie grecque. J'avais, en effet, traduit le Nouveau Testament en entier, lequel avait fait l'objet d'une 
publication à part, mais lorsqu'il s'agit alors de le traduire, ce fut le Père Mateos, professeur à l'Institut Biblique, qui s'en chargea. Tout ceci a constitué une expérience importante pour moi, nous nous sommes rendu compte que nous avions créé un style nouveau, bien que cet aspect de notre travail ait peut-être été exagéré par la suite. Nous avions introduit dans la Bible un vocabulaire ordinaire, courant, qui ne surprenait plus. On pouvait affirmer tranquillement que Jésus était sur une bourrique, on n'était plus obligé de dire un «âne ${ }^{4} \gg$. Il reste que, à un moment donné, certains sont allés jusqu'à écrire, par exemple : «pour en finir, il s'est produit...» «Dites donc, vous n'avez pas pu rester éveillés» ou «Comment avez-vous pu entrer à ce banquet sans tenue de soirée?». Personnellement, je pense que c'est aller trop loin.

Après cette période, je suis resté un peu en marge de la liturgie. En outre, j'étais allé en Amérique. Cependant, je crois que j'ai fait ce que j'ai pu dans le cadre du langage de l'Église, et que le résultat fut, pour moi, très important. Plus tard ce langage a encore changé; on le renouvelle constamment. Cependant, je crois que quelque chose de décisif a été acquis de façon définitive: la conscience de pouvoir utiliser une langue vivante. Il n'y a pas, en espagnol, de tradition biblique telle que la tradition anglaise et allemande, et, à ce moment-là, un grand effort a été fait en ce sens. Si nous comparons les traductions espagnoles et anglaises de la Bible, les espagnoles sont aujourd'hui en général meilleures, parce que les traductions anglaises gardent le «th» final et l'archaïsme de la langue biblique. En allemand, le problème ne se pose pas parce que la littérature allemande commence avec Luther, avec la traduction de la Bible faite par Luther, et que la prose de Luther s'inspire elle-même de la Bible. Pour moi, la prose de Luther est plus moderne que celle de Goethe (il se peut que dise là une hérésie. Si quelque professeur d'allemand me lit, il n'a qu'à ignorer cette ligne!).

J'ajouterais que, durant ma première période rilkéenne, $\mathrm{j}$ 'ai aussi traduit Hölderlin et que, en 1949, j'ai publié douze de ses poèmes. À ce moment-là, Hölderlin n'était pas à la mode comme il l'est devenu plus tard. En outre, j'ai traduit, non le Hölderlin fou, celui qui est à la mode, mais le Hölderlin encore en possession de toutes ses facultés, celui des grands hymnes, le bon Hölderlin. Par la suite, j'ai aussi traduit L'Archipel.

Vers 1960, une collection a été mise sur pied, les «Classiques Planeta» qui proposait de gros volumes sur papier bible. J'étais le directeur des sections anglaise, allemande et russe (j'ignore le russe, mais je me suis mis en quête de bons traducteurs) et alors, comme il est naturel, $j$ 'en ai profité et $j$ 'ai choisi de traduire moi-même ce qui me plaisait de traduire. J'ai traduit ainsi un volumineux tome de Goethe dans lequel se trouvaient le Faust I et II, dans la version métrique, Werther et quelques autres choses. Je dois avouer que je déteste Goethe, et, pour savoir si ce sentiment était justifié ou non, j'ai décidé de le traduire. Ma version métrique du Faust ne présente aucun problème - du moins, c'est ce que je crois - bien que la prose de cet auteur ne me plaise pas. Je crains de n'avoir pas été tout à fait honnête dans ce jeu, parce qu'en tant que traducteur littéral et objectif je peux parfois utiliser mon objectivité avec une certaine dose de mauvaises intentions; cependant, dans ce cas, toute la faute ne me revient pas. Allez-y voir et vous vous rendrez compte que Goethe écrit réellement ainsi, à cette différence près que la traduction rend un son un peu bizarre. Cependant, je crois, moi, que l'auteur écrivait réellement ainsi. J'ai traduit sans pitié, rendant compte de cette voix légèrement pédante et légèrement... je ne sais quoi dire, parce que n'importe quel autre adjectif paraîtrait irrévérencieux. Ce n'est pas de ma faute, sa prose est ainsi. En revanche, Faust, parce qu'il était en vers, me semble être meilleur.

J'ai aussi traduit Melville: Moby Dick et quelques autres choses en plus. Cela a été pour moi un plaisir parce que Melville est très agréable à traduire, encore que, à ce moment-là, j'aie commis l'erreur de le traduire avant d'avoir traduit Shakespeare, et il 
aurait mieux valu faire le contraire : commencer par Shakespeare pour ensuite trouver le ton qui domine chez Melville. Le problème avec Melville, qui par ailleurs écrit très bien, est que son style est toujours un peu parodique et, pour un traducteur, c'est un problème difficile: traduire la parodie est toujours difficile, puisqu'elle comprend la référence. En plus, j'y ai trouvé un problème technique. Heureusement, j'avais pu me procurer un dictionnaire multilingue des termes maritimes - de navigation à voile — que j'avais placé sur une table spéciale de grandes dimensions. Tout les termes qui y figuraient étaient expliqués dans cinq langues et accompagnés de dessins. J'avais, en outre, la traduction de Pavese qui je suppose - devait avoir bénéficié aussi de quelque aide technique, faute de quoi il est très difficile de faire ce travail. Le pire chez Melville, ce n'est pas le langage des cordages et autres technicalités, mais l'utilisation constante de la métonymie, de telle sorte que, lorsqu'il nomme les parties du bateau, il ne mentionne habituellement pas la partie en question, mais celle qui se trouve à côté. C'est quelque chose de vraiment peu commode pour un traducteur.

Je me suis attaqué ensuite à Shakespeare et j'ai traduit tout son théâtre, en prose naturellement, à l'exception des chansons qui, inévitablement, devaient être en vers, ce qui occasionne une grande perte. Avant que n'existent les «Classiques Planeta», j'avais tenté de traduire quelques tragédies de Shakespeare en vers blancs (c'est-à-dire en hendécasyllabes blancs - je n'allais tout de même pas faire des alexandrins !). Comme dans le texte traduit, ma traduction compte plus de syllabes; le procédé auquel je pouvais recourir était celui du chevauchement et des vers plus nombreux, c'était un travail très exigeant mais qui n'était pas irréalisable. Pour chaque ensemble de dix vers dans le «blank verse» de Shakespeare, je donnais douze, treize ou quatorze vers blancs en espagnol. C'était un travail énorme et, comme j'avais besoin d'aide, j'écrivis à une fondation quelconque en y envoyant des échantillons, un de Coriolan, un autre de Hamlet et enfin un du Roi Lear, chacune de ces scènes devant montrer ce qui pouvait être fait. Non seulement je ne reçus aucune aide, mais encore, cette même année, on accorda à l'un de mes amis espagnols qui habitait en Autriche - il savait très bien l'allemand - et à sa femme - qui était allemande - une bourse pour qu'ils traduisent un de mes recueils de poèmes. Le fait me parut extrêmement surprenant parce qu'évidemment la «somme» Shakespeare + moi-même est plus grande que la «somme» moi-même + ces amis.

Je mentionne ce fait pour que vous puissiez juger des bizarreries de l'histoire de la traduction.

Quand on traduit Shakespeare, plusieurs problèmes se présentent, l'un d'entre eux étant celui du style. Bien qu'il ressemble à celui du Siècle d'Or, le style de Shakespeare n'est pas le même. Quand on croit qu'il va être comme celui de Calderón, il ressort qu'il ne l'est pas, parce que Shakespeare évite toujours la perspective de face qui est celle du baroque; il est plutôt maniériste, il place les choses obliquement, non de face. À la manière baroque, nous aurions envie d'écrire que la lune est un «feu glacé», de même que «l'amour est une vie mortelle». En revanche, Shakespeare dit que la vie est un «feu pâle». C'est ça qui a servi à Nabokov plus tard dans le grand poème - roman qui porte ce nom. À ce sujet, la traduction de Un héros de notre temps faite par le fils de Nabokov (avec l'aval de l'auteur, son père) est un chef-d'œuvre de traduction, parce qu'on y explique tous les problèmes que l'on peut rencontrer, lorsqu'on traduit un roman russe romantique.

Le fait est que, dans Shakespeare, il y a un problème de style mais le plus grave est celui des jeux de mots qui, en plus, sont très mauvais et en général indécents. Quelquefois ça allait: j'avais presque toujours une solution mais dans d'autres cas ça n'allait pas. Alors, dans ma traduction, j'expliquais dans une note en bas de page le problème que posait l'original pour que le lecteur le comprenne. Je donnai un seul exemple de chaîne de pun, ou série de jeux de mots sur le même thème, comme ceux sont présents dans La mégère 
apprivoisée appelée en espagnol La fierecilla domada (la petite bête sauvage domptée) - bien que la bête sauvage n'y apparaisse aucunement, the shrew, qui appartient à la langue courante, est, en anglais, une femme furieuse. Il existe une métaphore, celle de la musaraigne - shrew —, mais comment écrire La musaraigne domptée? En outre, pour autant que je sache, la musaraigne n'est pas un animal très sauvage. C'est alors que commence la grande scène de l'affrontement entre Petruchio et Catalina, au cours de laquelle ils s'insultent mutuellement, mais, par jeu, comme il est fréquent dans le langage de Shakespeare. La seule chose qui intéressait Shakespeare était le langage, le reste lui était complètement indifférent il n'avait pas d'avis sur le monde, mais le langage, l'amusait. Les deux personnages se lancent donc des insultes. Dans l'original, il y en a quelques-unes qui sont en relation avec des noms d'insectes: d'abord apparaît be, jeu à partir de bee (abeille). De là, on passe à buzz, qui est bourdonner, et buzer, qui est pencher. Il y a aussi des oiseaux : interviennent le buzzand et la colombe, etc. J'ai dû pour ma part, tout modifier de la chaîne. À un moment, Petruchio dit:

- Ay buena Cata, yo te cargaré, pues sabiendo que eres joven y ligera...

Catalina: Demasiado ligera para que me alcance un moscón como tú, pero de mi peso, eso nada me quita.

Petruchio: Que no te quita, mosquita...

Catalina: Bien dicho, tú eres un moscón.

Petruchio: ¡Ah, mosquita de lento vuelo! ¿Te pillará un moscón?

Catalina: Si la mosquista no se amosca.

Petruchio: Vamos, vamos mosquita que pareces una avispa.

Catalina: Pues si soy una avispa, cuidado con mi aguijón.

Petruchio: ¿Quién sabe dónde tiene el aguijón une avispa?

Catalina : En la cola..

Petruchio: En la lengua...

Catalina: ¿En la lengua de quién? En la tuya, si hablas de colas ; así que adiós...

Petruchio: ¿Qué? Con tu lengua llevándote la cola.

Après cela, pendant mon séjour de dix ans au Canada, $\mathrm{j}$ 'ai fait trois traductions, dont je veux parler maintenant. L'une d'elles est l'Ulysse de Joyce. Il y en a une seconde que j'ai faite gratuitement pour ne pas perdre la main, et, parce que j'y prenais un véritable plaisir. C'est ce que j'ai fait de mieux dans toute ma vie de traducteur. Il s'agit de la traduction de Christian Morgenstern, poète allemand dont vous n'avez peut-être pas entendu parler mais que tous les Allemands citent en plaisanterie dans les conversations, car c'est un poète absurde. Il est mort en 1914. Il fait des jeux de mots qui sont de véritables satires métaphysiques. Morgenstern ne s'en prend à personne, il ne fait que jouer avec des situations absurdes. Dans son œuvre, il crée des personnages qui ont la caractéristique de ne pas exister. Deux d'entre eux sont des inventeurs et ils créent des choses bizarres. Par exemple, l'un invente une lampe diurne-nocturne qui fait l'obscurité, même s'il fait jour, quand on appuie sur l'interrupteur. L'autre invente une montre avec deux paires d'aiguilles, les unes vont d'avant en arrière et les autres d'arrière en avant, ce qui fait que le temps est aufgehoben. C'est un jeu très drôle pour qui connaît un peu la philosophie, parce qu'on dit, de cette manière, que le temps s'est dépassé lui-même. En allemand aufgehoben est un mot clé dans la philosophie de Hegel, lequel qui se félicite d'écrire en allemand, dans une langue si dialectique qu'elle possède le mot aufheben qui signifie des contraires: abolir et lever, assumer et dépasser. Là est contenue la philosophie de Hegel tout entière. Morgenstern s'en moque, disant que le temps se naaufgehoben avec une autre paire d'aiguilles qui vont à l'envers. Tout cela, je l'ai traduit en style Campoamor, comme il se devait. Campoamor a créé ce style de poésie qui a servi pour écrire des sortes de fausses fables que la génération de mes parents connaissait très bien. Ces fausses fables étaient très souvent 
indécentes, obscènes, d'autres fois simplement malodorantes. Cette forme a aussi un parallèle chez Morgenstern, mais ce dernier l'utilise dans un sens métaphysique.

J'ai fait aussi d'autres choses à cette époque, par exemple une traduction de Brecht qui n'est pas encore publiée pour des raisons de copyright. Ce travail m'a obligé à passer de Rilke à Brecht - un saut mortel - tout en revoyant Hölderlin. Brecht est le cynisme absolu, la brutalité et, en même temps, le langage parlé. Il y a parfois des chansons que j'ai traduites métriquement pour qu'elles puissent être chantées. Il y en a une que j'ai sur disque, interprétée par Brecht lui-même, et que j'ai traduite en suivant la musique de Kurt Weill dans L'opéra de quat'sous. Je ne peux hélas! la chanter ici.

Le langage de Brecht n'a rien à voir avec celui de Hölderlin, ni avec celui de Goethe, ni avec celui de Rilke. Un des sujets de Brecht est d'attaquer Goethe. La petite chanson dont je parle s'appelle «Chanson de l'insuffisance humaine». C'est évidemment une attaque féroce à l'endroit de la fin de Faust, quand les anges emportent au ciel le protagoniste en disant: «Nous pouvons sauver celui qui fait des efforts.» Dans la chanson, au contraire, il est dit que, l'homme aura beau faire des efforts, il ne réussira jamais à être sauvé, parce que, pour malin et malhonnête qu'il soit, il ne le sera jamais assez dans ce monde.

C'est aussi à cette époque que j'ai traduit Ulysse, traduction qui a connu un certain succès. Le travail a été facilité par le fait que j'avais traduit Shakespeare auparavant, car, dans Ulysse, il y a une impersonnalité toute shakespearienne dans de nombreuses voix (dans chaque chapitre, on entend une ou deux voix différentes). Pour un traducteur, c'est là un des efforts les plus difficiles qu'il doit faire: il faut changer de ton à chaque moment. Par exemple, il y a un chapitre, qui est en soi une petite histoire de la prose anglaise, dans lequel Joyce change de langage graduellement, allant de l'anglais médiéval jusqu'à son époque, contemporaine d'Oscar Wilde. Comment un traducteur peut-il résoudre ces problèmes dès lors qu'il n'a pas d'équivalents? Même l'auteur, qui a donné dans une lettre des pistes sur ses modèles, n'a pas tout explicité, et, très souvent, on ne sait pas très bien quel est le modèle anglais. Quelques-uns de ces parallèles, qui n'étaient pas dans la lettre de Joyce, je les ai reconnus moi-même mais, pour d'autres, je n'ai pas pu le faire, et c'est là que j'ai dû faire appel à toutes les ressources possibles, parce qu'il se produit continuellement un changement et qu'il n'y a jamais de voix personnelle.

Pour terminer, j'ai traduit moi aussi un peu de catalan. Avant de venir en Catalogne, j'avais appris le catalan en lisant, comme s'il s'agissait du grec, pour lire Maragall et Platon, parce que les traductions en castillan étaient abominables. Je me rendis compte alors que Maragall n'était pas dans les librairies de Madrid (pas plus d'ailleurs, que dans celles de Barcelone). J'ai donc dû chercher ses livres dans les bibliothèques et mettre au point une anthologie de Maragall soigneusement recopiée à la machine, qui devait — j'ai pu le constater ensuite - ressembler à toutes les anthologies parues depuis de cet auteur. J'en ai fait la traduction avec un dictionnaire, parce que je ne comprenais pas tous les mots (je ne savais qu'un peu de valencien pour avoir habité à Valence) et je respectais soigneusement la métrique. J'ai ainsi traduit entre autres choses, le Cant spiritual. Le temps passa et, à l'époque où j'étais au Canada, mon grand ami Gabriel Ferrater décédait à San Cugat. Sa mort survint quand une édition bilingue de la plupart de ses poèmes était en préparation sous le titre Mujeres y Días (Femmes et Jours). On m'écrivit pour que j'en traduise quelques-uns. Ce volume a été publié très tardivement et ce n'est qu'hier que j'ai reçu un petit article publié dans la revue Manresa Faig, où je parle de ces traductions. 


\section{DÉBAT}

Question: Vous avez dit qu' un chapitre d'Ulysse était un résumé de la prose anglaise. Pourriez-vous expliquer comment vous avez résolu ce problème en espagnol?

À l'oreille. J'ai essayé de faire quelque chose qui ressemble à l'original, c'est-à-dire qui soit plus ou moins archaïque en espagnol. Cependant, très souvent, l'archaïsme n'avait pas de modèle. Je ne pouvais utiliser l'arcipreste de Hita, par exemple, parce que cela ne correspondait à rien. J'ai essayé de faire une espèce de pastiche vague. Ce chapitre y perd beaucoup dans la traduction, surtout parce que le lecteur en espagnol ne peut reconnaître le modèle anglais et, même si l'on suppose qu'il connaît la littérature anglaise, ce serait très difficile qu'il la reconnaisse dans le texte espagnol. Dans d'autres cas, c'est plus facile. Quand Joyce imite le journal de Pepys, par exemple, ça ne se voit plus, il y a des trucs plus visibles. Au début, j'ai dit que traduire est toujours un échec et, dans ces cas-là, c'est encore plus vrai. Les échecs sont parfois majeurs, parfois mineurs.

Question: Au début vous avez parlé de la question de l'oreille quand on traduit. Pourriez-vous développer un peu plus cette idée?

Oui, la littérature est naturellement auditive. Quand nous lisons un auteur, nous l'écoutons, il a un ton, une musique et, ce qu'il faut tenter, c'est de produire une musique analogue dans sa propre langue. En général, la lecture est toujours un problème auditif. Si nous écoutons ce que nous lisons, nous ne le comprenons pas et il n'est possible ni de l'apprécier ni d'en jouir. Personnellement, je pense que, lorsque je lis dans une autre langue, je dois essayer d'entendre davantage le texte, sinon je perds le rythme, surtout quand il s'agit de l'allemand, qui a une syntaxe très différente de l'espagnol. La syntaxe anglaise est plus familière: je peux aller plus vite. En revanche, en allemand, il faut que je réfléchisse pour voir comment je vais emboîter le participe avec ce qui est avant, sans perdre la mélodie. Une historiette allemande raconte qu'un homme était au théâtre. À un moment donné, après le premier acte, il se souvint de quelque chose, ou bien il lui arriva quelque chose, alors, il voulut s'en aller à l'entracte, mais il ne put le faire car les participes n'apparaissaient qu'au deuxième acte.

Question: Vous utilisez le magnétophone pour traduire?

Non, jamais, parce que cela doit être simultané. Pour moi, traduire est simultané. Généralement je traduis à la machine, sauf quand c'est en vers. Traduire à la machine, en plus d'être conseillé d'un point de vue commercial, a l'avantage d'aider à maintenir la vitesse, et ça, c'est important pour traduire. C'est comme aller à bicyclette : si l'on va trop lentement, on tombe. On perd le rythme, la mélodie, et alors c'est impossible. Il est nécessaire d'avoir un peu de vitesse ou alors il n'y a pas moyen; plus on va vite, mieux c'est.

Question: Comment avez-vous résolu la question des traits dialectiques qui apparaissent dans le texte original?

Cela dépend du texte, de la manière que les emploie Joyce dans Ulysse; avec Shakespeare, j'ai eu aussi ce problème. Dans Henri $V$, j'ai eu beaucoup de mal, parce que certains personnages parlent en gallois, avec un accent gallois, et d'autres avec des accents encore différents. Ce que j'ai fait, c'est traduire celui qui avait l'accent gallois comme s'il était galicien, à l'autre j'ai prêté un accent plus conventionnel, disons par exemple, un accent de la Manche, pour que le lecteur comprenne que, dans le texte original, le personnage parlait avec des traits régionaux. C'est ce que j'ai tenté de faire parce qu'il me semblait que c'était l'équivalent. Cependant on ne peut pas donner de règles parce que chaque texte, chaque auteur, a son charme et qu'il faut essayer de l'imiter. 
Question: Vous ne croyez-vous pas que le pire qui puisse arriver à un traducteur, c'est de traduire un texte mal écrit?

C'est vrai. Le pire, c'est que, très souvent, le traducteur paie pour les péchés de l'auteur. Quand le texte est transposé dans une autre langue, cela ne pardonne pas. C'est ce qui m'est arrivé avec Henry James. Je l'ai traduit fidèlement et on a critiqué ma traduction; mais on n'y peut rien, il n'y a pas de solution. Moi, je l'ai traduit comme il était, que devais-je faire? Le limer? L'améliorer? Le réviser? Non, parce que le texte serait devenu autre chose. Dans la traduction, c'est le traducteur qui est ridicule et qui paie les pots cassés.

Question: Vous avez traduit Lawrence?

Non, heureusement, j'ai refusé. Dans le cas de Henry James, j'avais été imprudent. Je me souvenais vaguement de l'avoir lu dans le passé. Le grand Henry James! Puis, lorsqu'on le traduit, on se rend compte que c'est réellement catastrophique. Plus tard, un de mes amis, fin critique qui avait fait un compte rendu de cette traduction, m'a dit : «Tu as raison mais va donc dire à Henry James qu'il écrit mal !» Ceci est beaucoup plus fréquent dans les traductions techniques. Les textes techniques peuvent être bons, tout en étant absolument abominables. J'en suis venu parfois, à refuser de signer certaines traductions simplement parce que, m'étant engagé auprès de l'éditeur à la faire, je ne voulais pas me rendre responsable de ce qui ne pouvait être que mauvais. Il s'agit alors d'utiliser un pseudonyme et l'affaire est réglée.

Question: Vous avez dit que vos traductions étaient très littérales, mais pourriezvous expliquer ce que vous faites quand vous trouvez un mot dont l'image n'est pas la même dans les deux langues? Ce que vous faites pour que le lecteur saisisse les différentes connotations d'un mot dans une langue ou dans une autre?

J'essaie de dire ce que dit l'original. Souvent cela se perd, surtout quand il y a des jeux de mots (j'ai dit déjà comment j'essaie de me débrouiller quand il y a un jeu de mots essentiel). Alors, si ça marche, ça marche et, sinon, je me résigne, car on ne peut pas dire au lecteur: «Voyez, l'original dit ceci.» Très souvent on y perd beaucoup; d'autre part, il y a des références sociales, politiques, historiques qui disparaissent inévitablement. Si j'en ai la possibilité, j'ajoute une note en bas de page, mais, si je ne peux pas le faire, le sens originel se perd.

Question: Quand vous traduisez de la poésie, quels sont les éléments poétiques que vous conservez prioritairement : rime, rythme, mètre? J'ai constaté que quand vous avez traduit Morgenstern vous avez conservé la rime.

Je conserve tout, absolument tout, quelquefois j'ai même enrichi. Je vais vous citer une autre poésie très drôle pour que vous vous rendiez compte que quelque fois il faut changer. Je donnerai d'abord la traduction et ensuite le texte original.

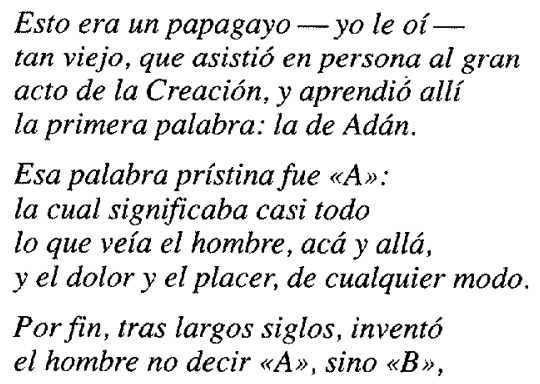


y tras de añadir «L» y gritar «O $O$, hasta la Zeda poco a poco fue.

El sabio papagayo, mientras tanto, se hizo más viejo que Matusalén, conservando en su pecho y en su canto la primera palabra del Edén.

Al fin también un día él hincó el pico, pero guarda su imagen de escayola, con une «A» de material muy rico, la Asociación Lingüistica Española.

Naturellement l'association linguistique espagnole ne se trouve pas dans l'original Der Papagei :

\author{
Es war einmal ein Papagei, \\ der war beim Schöpfungsakt dabei \\ und lernte gleich am rechten Ort \\ des ersten Menschen erstes Wort. \\ Des Menschen erstes Wort war A \\ und hiess fast alles, was er sah, \\ z.B. Fisch, z.B.Brot, \\ z.B. Leben oder Tod.
}

Erst nach Jahrhunderten voll Schnee erfand der Mensch zum $A$ das $B$ und dann das $L$ und dann das $Q$ und schliesslich noch das $Z$ dazu.

Gedachter Papagei indem ward älter als Methusalem, bewahrend treu in Brust und Schnabel die erste menschliche Vokabel.

Zum Schlusse starb auch er am Zips. Doch heut noch steht sein bild in Gips, geschmückt mit einem grünen A, im Staatsschatz zu Ekbatana.

Là où le texte original dit «El tesoro de Ekbatana», j'ai mis l'association linguistique espagnole, parce que c'était beaucoup mieux, et, en plus, parce que Gips renvoie à du plâtre (escayola), ce qui me donnait la rime en $D a$ de la strophe. Ensuite, quand le texte original dit, «starb auch er am Zips», qui est plutôt de la langue parlée, j'ai trouvé un jeu de mots tout fait: «Al fin también un día, él hincó el picó» (enfin un jour, il piqua $d u n e z^{5}$ ), c'est-à-dire il mourut (il tomba). Mais pour un perroquet, c'est formidable: quand un perroquet meurt, il pique réellement du nez (hincar el pico). Je me suis donc permis toute une série de petites adaptations - mon mètre est l'hendécasyllabe, alors qu'en allemand çe n'est pas celui choisi. Malgré cela, je crois qu'il ne pouvait y avoir de traduction plus littérale.

Question: Pourriez-vous expliquer le problème de la métonymie auquel vous avez fait allusion quand il est question de la traduction de Moby Dick?

J'ai respecté la métonymie: je ne pouvais pas la corriger, précisément parce que c'est un truc, une manie de l'auteur et, les manies, il faut les respecter parce qu'elles font partie de sa conception de la littérature. C'était un homme qui avait beaucoup navigué dans 
des baleinières et il portait en lui le langage au point de pouvoir se permettre la coquetterie de ne pas dire les choses directement mais seulement indirectement. Pour le lecteur actuel, ça n'a pas d'importance. Ça lui est égal que l'on dise un cordage, un hauban ou un baupré, mais tant qu'à faire, respectons le jeu.

Question: Quand on fait la traduction d'un livre pour lequel il existe des versions antérieures, jusqu'à quel point convient-il de comparer les autres traductions?

C'est toujours une référence. Quand j'ai traduit Ulysse, j'avais installé un appuielivres articulé en cinq parties; dans la partie du milieu, se trouvait l'original d'un côté, une traduction antérieure, très bizarre, inégale, mais pas complètement mauvaise, parce qu'il y avait parfois des réussites. Elle avait été faite en Argentine, dans de mauvaises conditions, il y a très longtemps. On m'a raconté que Borges avait dit qu'il la ferait, mais, naturellement, il ne l'avait pas faite, et alors, c'est une autre personne à qui l'on avait eu recours. D'un autre côté de l'appuie-livres, il y avait la traduction italienne et également une traduction allemande, celle de Goyen, qui est classique - bien qu'elle soit aujourd'hui dépassée - et, enfin, il y avait la française, qui est de la folie, parce qu'elle avait été faite en équipe et que Joyce y avait travaillé. C'est lui le responsable du désastre. Joyce, évidemment très prétentieux en matière linguistique, connaissait beaucoup de mots français et il a voulu le montrer et employer tout le jargon ${ }^{6}$ français. Le résultat fut catastrophique. Il est curieux de constater que très souvent, on considère comme une garantie de valeur de la traduction française, ce qui, précisément, lui fait perdre cette valeur. Joyce aurait dû laisser les Français tranquilles et ceux-ci se seraient mieux arrangés. Au moins, le résultat aurait été du français.

Question: Est-ce vrai que le traducteur ne doit pas avoir d'opinions littéraires et accepter toutes les conceptions?

La seule chose que doit faire le traducteur, c'est essayer de choisir ce qu'il aime le plus - s'il le peut - et à l'intérieur de ces limites, se débrouiller. Cela dit, sa conception de la traduction ne doit pas être une conception, mais une habitude, une coutume. Il faut s'entraîner. Pour être clown, pardon de faire cette comparaison, il faut s'entraîner, et, pour être traducteur aussi, mais il y a de ça.

Question : Croyez-vous que pour traduire il faille avoir une formation universitaire?

Une formation universitaire, non. Ce qu'il faut c'est une culture littéraire. Il y a des gens qui ont une grande culture littéraire et qui ne sont pas passés par l'université ou qui sont passés par une faculté de sciences. Gabriel Ferrater n'a jamais été licencié en philologie (la philologie «anglaise et allemande» de cette époque): il ne réussissait jamais ses examens. Il avait déjà traduit plusieurs livres de l'allemand et il ratait ses examens parce qu'il ne savait pas faire ce qu'on lui demandait, qui était un thème ou quelque chose d'approchant. Il a aussi raté son examen d'histoire. Quand il s'y est présenté, c'est sa culture littéraire qui l'a perdu. Le professeur lui posa des questions sur saint Louis, le roi de France, et il commença à parler des Chroniques de Joinville où il est question de ce roi. Le professeur, qui ne connaissait pas les Chroniques, le recala.

Question: Que vouliez-vous dire quand vous avez dit que la prose allemande était assez problématique et que vous aimiez mieux la prose anglaise?

Nietzsche disait que Heine et lui-même étaient les meilleurs écrivains en prose de langue allemande et, personnellement, je suis tout à fait d'accord, mais les Allemands ne partagent pas tous cette opinion. Si on pose la question aux Allemands, ils répondent que le meilleur écrivain en prose est Goethe, et alors, c'est à mon tour de ne plus être du tout 
d'accord. En outre, la prose allemande actuelle est trop influencée par les universités, par leur langage artificiel, et elle a en plus un problème de traits dialectaux. Brecht, par exemple, a dû, dans son théâtre, faire des miracles, parce que la question est la suivante : comment créer un théâtre réaliste qui sonne bien dans toutes les régions allemandes.

L'anglais est une langue qui a la chance d'avoir une tradition de prose merveilleuse, la meilleure sur laquelle je me sois penché - meilleure que le français. En français, il y a certaines coupes, une tradition limitatrice qui ne fait pas bon ménage avec la langue parlée. En ce sens, réellement, 1'histoire de la littérature anglaise est exemplaire, elle a toujours eu de bonnes relations avec la vie.

Question: Croyez-vous que traduire un auteur ou certains livres d'un auteur peut avoir une influence sur la langue poétique d'un pays?

Évidemment, beaucoup! Les poètes qui traduisent ou, s'ils ne traduisent pas, qui lisent en langue étrangère sont le lieu d'une sorte d'écho. Pour autant que je me souvienne, Rubén Darío n'a pas traduit, mais il a écrit des vers en français. Ce fut une greffe de la poésie symboliste et parnassienne française sur l'espagnol. La traduction, dans ce cas, n'était pas une traduction de textes précis, mais celle de tout un système, de toute une langue et de toute une série de techniques.

En 1930, Luis Cernuda avait un ami appelé Gebser, hispaniste qui a écrit un livre sur Rilke en Espagne. Cet homme l'aida à lire Hölderlin et lui en fít même quelques traductions. La conséquence en est Invocaciones a la gracia del mundo et Las nubes qui, à leur tour, rendirent possible l'existence de choses comme Sombras del paraíso de Vicente Aleixandre. C'est une histoire assez difficile à expliquer, normalement, les professeurs et je dis du mal des professeurs, j'en ai le droit, je suis du métier - ne la racontent pas volontiers, car cette histoire est, sans doute, souterraine, secrète. Il s'avère qu'à un moment donné quelqu'un a lu quelque chose d'une autre personne et que le fait a changé le panorama, mais qu'il n'a pas été consigné. En conséquence, il n'apparaîtra pas dans les histoires de la littérature.

\section{(Traduction de Brigitte Lépinette)}

\section{Notes}

1. Conférence prononcée à l'École universitaire de traducteurs et interprètes de l'Université autonome de Barcelone en mai 1982. Une première version a été publiée en espagnol dans Cuadernos de Traducción e Interpretación / Quaderns de Traducció i Interpretació, $\mathrm{n}^{\circ} 2$, 1983. Ces réflexions, faites par l'un des grands traducteurs espagnols actuels, méritaient évidemment d'être traduites et d'avoir une large diffusion. Nous y ajoutons une traduction du débat qui suivit, car il nous a paru extrêmement intéressant.

2. N.d.T. Le pronom personnel complément masculin peut être, selon les régions, lo ou le.

3. N.d.T. L'espagnol de l'Amérique du Sud emploie pour «vous» «Ustedes», alors que l'espagnol de la Péninsule a recours à la forme «vosotros».

4. N.d.T. Le terme asno (âne) appartient à un registre élevé en espagnol alors que burro (bourrique) ne l'est pas.

5. Exemple des difficultés de traduction, Hincar el pico en espagnol se traduirait littéralement par «piquer le bec» ou «piquer du bec» (conservant l'empreinte de hincar el diente - commencer quelque chose) et non «piquer du nez», comme je l'ai traduit, mais piquer du nez (signifiant s'endormir quand on est assis) existe en français comme expression figée et conserve l'image de la chute inconsciente, tandis que piquer du bec n'est pas une expression figée et ne peut avoir un sens que littéral. Bref, en tant que traductrice de traducteurs, je m'accuse de ce demi-échec. On me concèdera cependant qu'un jeu de mots se situant au carrefour de trois langues (l'allemand de l'original, l'espagnol du traducteur et mon français) ne rend pas le travail aisé...

6. En français dans le texte original. 